\title{
Effect of synthetic polymers on tRNA nanoparticle formation
}

\begin{abstract}
In this review, we have examined the effects of several synthetic nanoparticles such as poly (ethylene glycol) PEG-(PEG-3350, PEG-6000), methoxypoly (ethylene glycol) anthracene (mPEG-anthracene), methoxypoly (ethylene glycol) poly (amidoamine) (mPEG-PAMAM-G3), (mPEG-PAMAM-G4) and poly (amidoamine) (PAMAM-G4) on tRNA structure and dynamics. Atomic force microscopic (AFM) images and spectroscopic data were analysed and the effects of synthetic polymer complexation on tRNA stability, aggregation and particle formation are discussed. Hydrophilic and hydrophobic contacts were dominated in the polymer-tRNA complexation and the overall binding constants showed that the order of binding is PEG-6000>PAMAMG4>PEG-3350>mPEG-PAMAM-G4>mPEG-PAMAM-G3>mPEG-anthracene. The morphology of polymer-tRNA complexes showed major aggregation and nanoparticle formation of tRNA, in the presence of synthetic nanoparticles.
\end{abstract}

Volume 2 Issue 3 - 2015

\author{
Tajmir-Riahi HA,' Bekale L, I Kreplak L² \\ 'Department of Chemistry-Physics, University of Quebec, \\ Canada \\ ${ }^{2}$ Department of Physics, Dalhousie University, Canada
}

\begin{abstract}
Correspondence:Tajmir-Riahi HA, Department of ChemistryPhysics, University of Quebec at Trois-Rivieres, C.P. 500, TroisRivieres (Quebec), G9A 5H7, Canada, Tel 819-376-50II, Fax 819-376-5084 Email tajmarri@uqtr.ca
\end{abstract}

Received: April 21, 20I5 | Published: June 06, 2015

Keywords: PEG, Dendrimer, tRNA, Particle formation, Spectroscopy, AFM

Abbreviations:PEG, Poly (Ethylene Glycol); mPEG, Methoxypoly (Ethylene Glycol); PAMAM, Poly (Amidoamine); CD, Circular Dichroism; FTIR, Fourier Transform Infrared; AFM, Atomic Force Microscopy

\section{Introduction}

Synthetic polymers play a major role in therapeutic drug, protein and gene delivery systems. ${ }^{1-6}$ Among synthetic polymers (Scheme 1), poly (ethylene glycol) and its derivatives show potential applications in gene and drug delivery due to their solubility, nontoxicity and biocompatibility. ${ }^{1-3}$ Dendrimers (Scheme 1) are also unique synthetic macromolecules of nanometer dimensions with a highly branched structure and globular shape with strong affinity towards DNA and RNA complexation ${ }^{4-10}$ It has been shown that synthetic polymers induce significant changes in DNA and RNA solubility and structure under given conditions. ${ }^{8-20}$ Synthetic polymers are also used to transport miRNA and siRNA in vitro.$^{11-24}$ PEGylation of synthetic polymers such as dendrimers is shown to reduce toxicity and increase biocompatibility and DNA Transfection. ${ }^{6,7,9}$ It is well demonstrated that synthetic polymers induce DNA and RNA aggregation and particle formation. ${ }^{25-29}$ However, not much of investigation on the effect of synthetic polymers on tRNA structure and dynamic has been reported. Therefore, it was of interest to review and compare the effects of several synthetic polymers on tRNA aggregation and particle formation that are reported in the literature.$^{25,26}$

In the following section we compare the binding of tRNA to several synthetic polymers such as PEG-3350, PEG-6000 and mPEG-anthracene, mPEG-PAMA-G3, mPEG-PAMAM-G4 and PAMAM-G4 at physiological conditions. The data obtained from multiple spectroscopic measurements and AFM microscopic images will be analysed and the effects of various synthetic polymers on tRNA aggregation and particle formation are reported here.

\section{AFM images and morphology of synthetic polymer- tRNA complexes}

Major tRNA aggregation and particle formation were observed in the presence of PEG, mPEG-anthracene, dendrimers and PEgylated dendrimers. ${ }^{25,26}$ In the case of PEG-3350, PEG-6000 and mPEG- anthracene, the mica surface was covered by small complexes of various shapes (Figure 1-panel A). In each case the average height and average volume of the complexes were estimated. The PEG3350 complexes had an average height of $0.75 \pm 0.12 \mathrm{~nm}(\mathrm{n}=143)$ and an average volume of $61 \mathrm{~nm}^{3}$ (Figure 1-panel A). PEG-6000 complexes were doubled in height compared to the PEG-3350 ones, average height of $1.3 \pm 0.3 \mathrm{~nm}(\mathrm{n}=208)$ and average volume of 520 $\mathrm{nm}^{3}$ (Figure 1-panel A). Finally, mPEG-Anthracene complexes had an average height of $0.59 \pm 0.2 \mathrm{~nm}(\mathrm{n}=108)$ and an average volume of 252 $\mathrm{nm}^{3}$ (Figure 1-panel A). Assuming a molecular weight of $27 \mathrm{kDa}$ and a density of $1 \mathrm{~g} / \mathrm{cm} 3$, each tRNA molecule should occupy a volume of $45 \mathrm{~nm}^{3}$. Hence the PEG-3350 complexes contain in average one tRNA molecules, the anthracene complexes five molecules and the PEG-6000 complexes ten molecules (Figure 1-panel A). The tRNA aggregation and particle formation in the presence of PEG and mPEG-anthracene were very similar to those of PEG-DNA complexes recently reported..$^{21,22}$

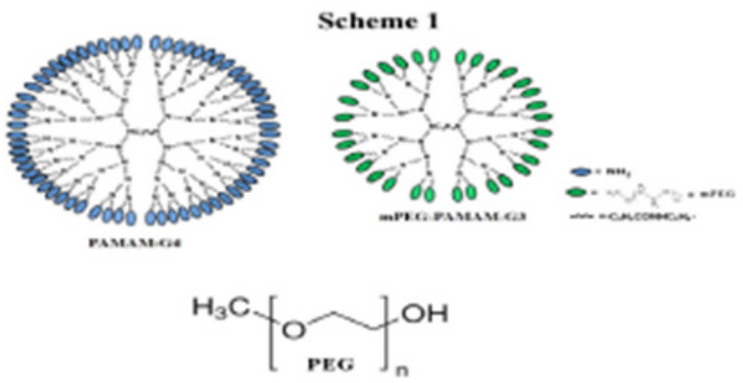

Scheme I Chemical structures of PEG, mPEG-PAMAM-G3 and PAMAM-G4.

Similarly in the cases of dendrimers and PEGylated dendrimers, the mica surface was covered by a layer that attributed to a mixture of free dendrimer, free pegylated dendrimers and free tRNA (Figure 1-panel B). On the top of this layer several round aggregates were observed (Figure 1-panel B). In each case the average height and average volume of the aggregates were measured. The mPEGPAMAM-G3 complexes had an average height of $0.29 \pm 0.18 \mathrm{~nm}$ $(\mathrm{n}=50)$ and an average volume of $80 \mathrm{~nm}^{3}$ (Figure 1-panel B). The mPEG-PAMAM-G4 complexes had an average height of $0.54 \pm 0.35$ $\mathrm{nm}(\mathrm{n}=50)$ and an average volume of $104 \mathrm{~nm}^{3}$ (Figure 1-panel B). Finally, PAMAM G4 complexes showed the best defined aggregates 
with an average height of $0.43 \pm 0.13 \mathrm{~nm}(\mathrm{n}=100)$ and an average volume of $102 \mathrm{~nm}^{3}$ (Figure 1-panel B). As one can see major aggregation and particle formation of tRNA were observed in the presence of these synthetic polymers, particularly in the cases of PEG-6000 and PAMAM-G4 nanoparticles (Figure 1-panel A \& B).
A
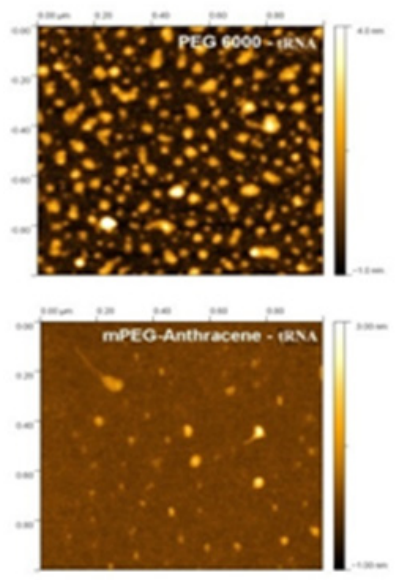

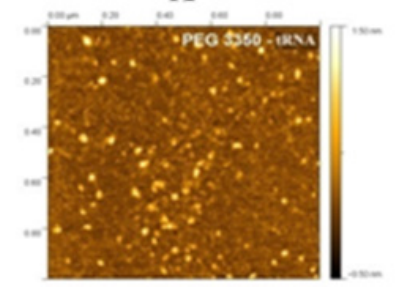

B

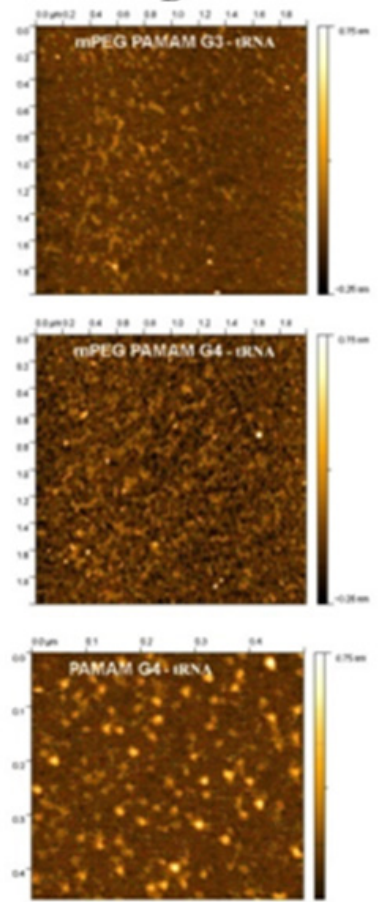

Figure I Tapping mode AFM images in air of synthetic polymer-tRNA complexes diluted 10 or 100 times in ultrapure water and adsorbed to mica. In all three cases, the surface was covered with aggregates. Panel A) complexes with PEG 3350, PEG 6000 and mPEG-anthracene and panel B) for complexes with mPEG-PAMAM-G3, mPEG-PAMAM-G4 and PAMAM-G4.

\section{Binding sites of synthetic polymers with tRNA}

FTIR spectroscopy is widely used to characterize the binding of ligand to DNA and RNA. ${ }^{25-29}$ Synthetic polymer complexes with DNA and tRNA via hydrophilic, hydrophobic contacts, groove binding and phosphate interaction. ${ }^{25-29}$ The infrared spectra and difference spectra of the free tRNA showed major alterations of tRNA in-plane vibrations and the backbone phosphate asymmetric and symmetric stretching bands. ${ }^{30-35}$ upon polymer complexation (Figure 2-panels A and B). Low concentration $(0.125 \mathrm{mM})$ of synthetic polymers PEG3350, PEG-6000, mPEG-PAMAM-G3, mPEG-PAMAM-G4 and PAMAM-G4 induced minor changes of tRNA vibrational frequencies, while at high polymer content $(1 \mathrm{mM})$ major alterations of tRNA in-plane and the backbone vibrational frequencies (Figure 2-panels $\mathrm{A}$ and B). The major intensity increases were associated with the guanine at 1698 (guanine N7), uracil at 1660 (uracil $\mathrm{O}_{2}$ and adenine at $1609 \mathrm{~cm}^{-1}$ (adenine N7) in the difference spectra of PEG-3350, PEG6000, mPEG-anthracene, mPEG-PAMAM-G3, mPEG-PAMAM-G4 and PAMAM-G4 complexes of tRNA (Figure 2-panels A and B, diff., $1 \mathrm{mM})$. The observed intensity changes were attributed to polymer interactions with tRNA guanine $\mathrm{N} 7$, uracil $\mathrm{O}_{2}$ and adenine $\mathrm{N} 7$ sites. ${ }^{25,27}$ Similarly, increase in the intensity of the backbone $\mathrm{PO}_{2}$ groups at 1241 (asymmetric $\mathrm{PO}_{2}$ ) and $1085 \mathrm{~cm}^{-1}$ (symmetric $\mathrm{PO}_{2}$ vibrations) were observed due to synthetic polymer- $\mathrm{PO}_{2}$ interaction (Figure 2-panels $A$ and B, diff., $1 \mathrm{mM})$.

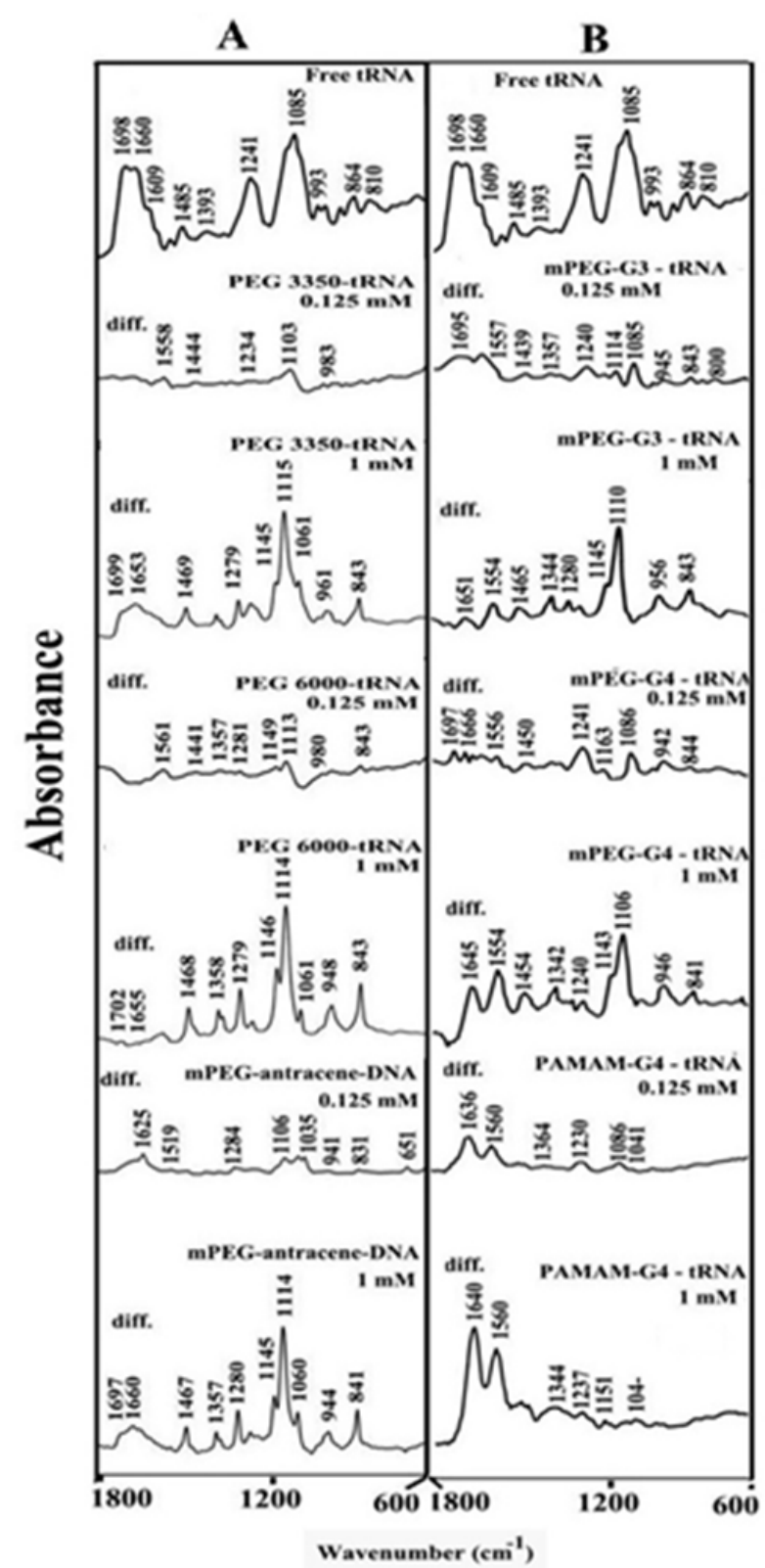

Figure 2 FTIR spectra and difference spectra [(tRNA solution + polymer solution) -(tRNA solution)] in the region of $1800-600 \mathrm{~cm}^{-1}$ for the free tRNA and its synthetic polymer complexes with PEG-3350, PEG-6000 and mPEGanthracene (panel $A$ ) and for mPEG-PAMAM-G3, mPEG-PAMAM-G4 and PAMAM-G4 (panel B) in aqueous solution at $\mathrm{pH} 7.3$ with various polymer concentrations $(0.125$ and I $\mathrm{mM}$ ) and constant tRNA content (I $2.5 \mathrm{mM})$.

\section{Hydrophilic and hydrophobic contacts in polymer- tRNA complexes}

A major shifting of the $\mathrm{OH}$ stretching of the free PEG at about $3430 \mathrm{~cm}^{-1}$ to a lower frequency in the infrared spectra of PEG-tRNA complexes was attributed to the hydrophilic interaction between PEG and tRNA polar groups. Similarly, the shifting of the NH stretching vibration at $3280 \mathrm{~cm}^{-1}$ in the spectra of the free dendrimer and pegylated dendrimers was due to the hydrophilic contacts between dendrimers terminal $\mathrm{NH}_{2}$ groups and the tRNA polar groups. ${ }^{25,27}$ However, hydrophobic interactions between tRNA and synthetic polymer were characterized by the shifting of the polymer antisymmetric and 
symmetric $\mathrm{CH}_{2}$ stretching vibrations, in the region of $3000-2800 \mathrm{~cm}^{-1}$. The $\mathrm{CH}_{2}$ bands of the free PEG at 3000, 2990, $2940 \mathrm{~cm}^{-1}$ exhibited a minor shifting, while the $\mathrm{CH}_{2}$ vibrations related to mPEGPAMAM-G3 located at 2946, 2884 and $2859 \mathrm{~cm}^{-1}$; for free mPEGPAMAM-G4 at 2942, 2876 and $2856 \mathrm{~cm}^{-1}$ and free PAMAM-G4 at 2969,2940 and $2834 \mathrm{~cm}^{-1}$ shifted to higher frequencies in the spectra of dendrimer-tRNA complexes. The shifting of the polymer antisymmetric and symmetric $\mathrm{CH}_{2}$ stretching vibrations in the region $3000-2800 \mathrm{~cm}^{-1}$ of the infrared spectra suggests the presence of minor hydrophobic interactions via dendrimer hydrophobic cavities and tRNA hydrophobic groups. ${ }^{25,27}$

\section{Synthetic polymer and tRNA conformation}

The CD spectra of tRNA and its synthetic polymer complexes are shown in Figure 3. The CD of the free tRNA is composed of four major peaks at 209 (negative), 221 (positive), 240 (negative) and 269 $\mathrm{nm}$ (positive) (Figure 3). This is consistent with CD spectra of double helical RNA in A conformation. ${ }^{36,37}$ As polymer complexes formed, a major increase in molar ellipticity of the band at $209 \mathrm{~nm}$ occurred and the amplitude of the band at 240 was reduced, while the intensity of the band at 269 decreased at high polymer concentration (Figure 3, panel A). However, no major shifting was observed for the band at $269 \mathrm{~nm}$ in the spectra of polymer-tRNA complexes (Figure 3, panel $\mathrm{A} \& \mathrm{~B})$. This is due to the presence of tRNA in A-conformation both in the free state and in the synthetic polymer-RNA complexes. This is also consistent with the infrared results that showed free tRNA in A-conformation with IR marker bands at $1698(\mathrm{G}), 1241\left(\mathrm{PO}_{2}\right), 864$ and $810 \mathrm{~cm}^{-1}$ (ribose-phosphate) with no major shifting of these bands in the polymer-tRNA complexes (Figure 2, panels A and B).

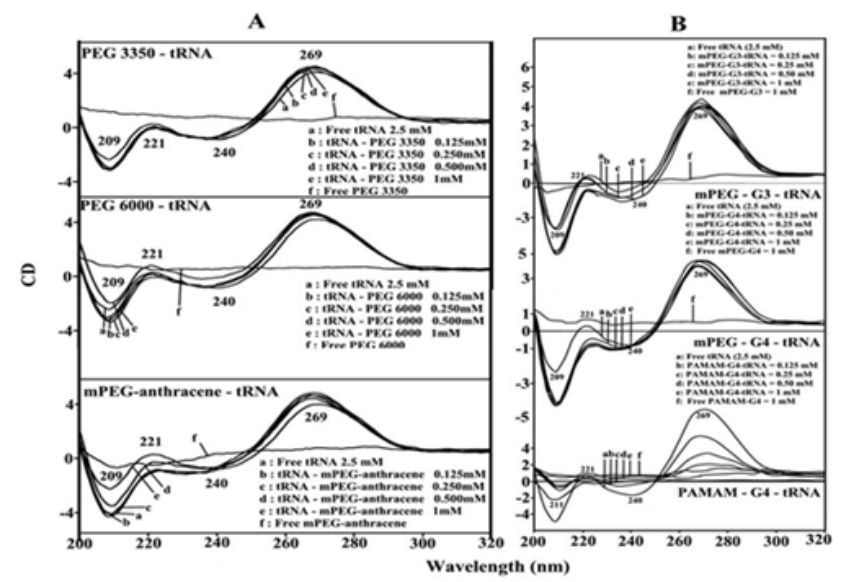

Figure $3 \mathrm{CD}$ spectra of tRNA in Tris- $\mathrm{HCl}(\mathrm{pH} \sim 7.3)$ at $25{ }^{\circ} \mathrm{C}(2.5 \mathrm{mM})$ with PEG-3350, PEG-6000 and mPEG-anthracene (panel A) and mPEGPAMAM-G3, mPEG-PAMAM-G4 and PAMAM-G4 (panel B) with 0.125, 0.25, 0.5 and I mM polymer concentrations.

The reduced intensity of the band at $269 \mathrm{~nm}$, in the spectra of polymer-tRNA complexes together with the major intensity changes of the band at 209 and $221 \mathrm{~nm}$ were attributed to the aggregation and particle formation of tRNA, in the presence of PEG-3350, PEG-6000, mPEG-anthracene (Figure 3, panel A) and mPEG-PAMAM-G3, mPEG-PAMAM-G4 and PAMAM-G4 (Figure 3, panel B). The extent of decrease of intensity was much pronounced in the case of PAMAM-G4 nanoparticle, where tRNA aggregation and particle formation were observed (Figure 3, panel B). This is consistent with AFM images of the synthetic polymer-tRNA complexes that showed major tRNA aggregation and particle formation by PAMAM-G4 nanoparticles (Figure 1, panel B).

\section{Stability of synthetic polymer-tRNA complexes}

The calculated binding constants of synthetic polymer-tRNA complexes by UV-visible spectroscopic method. ${ }^{38}$ showed $\mathrm{K}_{\mathrm{PEG}} 3350$ ${ }_{\text {tRNA }}=1.9 \times 10^{4} \mathrm{M}^{-1}, \mathrm{~K}_{\mathrm{PEG} 6000-\mathrm{tRNA}}=8.9 \times 10^{4} \mathrm{M}^{-1}$ and $\mathrm{K}_{\text {mPEG-anthracene }}=1.2 \mathrm{x}$ $10^{3} \mathrm{M}^{-1}, \mathrm{~K}_{\text {mPEG-G3 }}=7.6 \times 10^{-3} \mathrm{M}^{-1}, \mathrm{~K}_{\text {mPEG-G4 }}=1.5 \times 10^{4} \mathrm{M}^{-1}$ and $\mathrm{K}_{\text {PAMAM-G4 }}=$ $5.3 \times 10^{4} \mathrm{M}^{-1}$ (Figure 4, panels A\&B). ${ }^{25-27}$ Stronger polymer-tRNA complexation formed by PEG-6000 than PEG-3350 and mPEGanthracene, while PAMAM-G4 forms more stable complexes with tRNA than those of PEGylated dendrimers with the order of binding PEG-6000>PAMAM-G4>PEG-3350>mPEG-PAMAM-G4>mPEGPAMAM-G3>mPEG-anthracene (Figure 4, panels A\&B). ${ }^{25-27}$ This is indicative of PEG forms stronger complexes than $\mathrm{mPEG}$, dendrimers and PEGylated dendrimers. Similarly, stronger complexes form with larger PEG than smaller polymer. This is also consistent with the conclusion that synthetic polymer-tRNA interaction is more hydrophilic than hydrophobic occurred between polymer and tRNA polar groups. This conclusion can be supported by the argue that PEG with mostly hydrophilic character forms stronger complexes with tRNA, while mPEG-anthracene, with mostly hydrophobic nature forms weaker tRNA complexes. Similarly, PAMAM-G4 which has more cationic $\mathrm{NH}_{2}$ groups $\left(64 \mathrm{NH}_{2}\right.$ groups) than those of mPEG-PAMAM-G4 (32 $\mathrm{NH}_{2}$ groups) and mPEG-PAMAM-G-3 (8 $\mathrm{NH}_{2}$ groups) forms stronger complexes than PEGylated dendrimers (Figure 4, panels A\&B) [39,40]. The results showed that hydrophilic interaction is a major part of synthetic polymer-tRNA complexation.

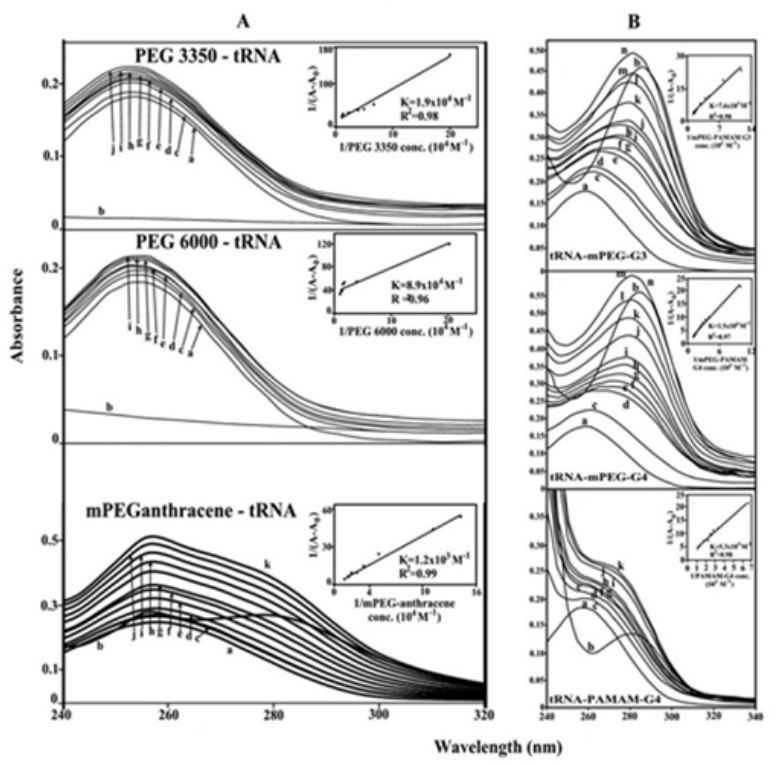

Figure 4 UV-visible results of tRNA and its PEG-3350, PEG-6000 (B) and mPEG-anthracene complexes (panel A) and for mPEG-PAMAM-G3, mPEGPAMAM-G4 and $\mu$ M PAMAM-G4 complexes (panel B) with free tRNA (I00 $\mu \mathrm{M})$; b) free polymer (I00); titrated with polymer (5 to $80 \mu \mathrm{M})$. Plot of $\mathrm{I} /$ (A-A0) vs (I/polymer concentration) for $K$ calculation of polymer and tRNA complexes, where $A 0$ is the initial absorbance of tRNA $(260 \mathrm{~nm})$ and $A$ is the recorded absorbance $(260 \mathrm{~nm})$ at different polymer concentrations $(5 \mu \mathrm{M}$ to $80 \mu \mathrm{M}$ ) with constant tRNA concentration of $100 \mu \mathrm{M}$ at $\mathrm{pH} 7.3$.

\section{Fluorescence spectra of synthetic polymer-tRNA and the number of binding sites}

As tRNA is a weak fluorophore, the titration of mPEG-anthracene was done against various tRNA concentrations, using mPEGanthracene excitation at 330-350 nm and emission at 400-450 nm. ${ }^{41,42}$ When mPEG-anthracene interacts with tRNA, fluorescence may 
change depending on the impact of such interaction on the mPEGanthracene conformation or via direct quenching effect. ${ }^{43}$ The decrease of fluorescence intensity of mPEG-anthracene has been monitored at $420 \mathrm{~nm}$ for mPEG-anthracence-RNA systems. The plot of $\mathrm{F}_{0} /\left(\mathrm{F}_{0}-\right.$ F) vs $1 /$ [tRNA] is shown in Figure 5A. Assuming that the observed changes in fluorescence come from the interaction between mPEGanthracene and polynucleotides, the quenching constant can be taken as the binding constant of the complex formation. The binding constant obtained was $\mathrm{K}_{\text {mPEG-anthracene-DNA }}=8.2( \pm 1) \times 10^{3} \mathrm{M}^{-1}$ (Figure 5A'). The association constant calculated for the $\mathrm{mPEG}$-anthracene-RNA adduct suggests low affinity mPEG-anthracene-tRNA, which is consistent with the UV results discussed above. The $f$ values obtained in Figure 5 , suggest that tRNA also interacts with fluorophore via hydrophobic interactions, which is consistent with our infrared spectroscopic results discussed (hydrophilic and hydrophobic contacts).

The number of binding sites occupied by mPEG-anthracene molecule on tRNA $(n)$ was calculated from $\log \left[\left(\mathrm{F}_{0}-\mathrm{F}\right) / \mathrm{F}\right]=\log \mathrm{K}_{\mathrm{S}}+$ $\mathrm{n} \log$ [tRNA] for the static quenching ${ }^{44-49}$ The linear plot of $\log \left[\left(\mathrm{F}_{0}-\right.\right.$ $\mathrm{F}] / \mathrm{F}]$ as a function of $\log [\mathrm{RNA}]$ is shown in Figure $5 \mathrm{~A}$ ". The $n$ values from the slope of the straight line was 1.3 for mPEG-anthracene-tRNA adduct (Figure 5A"). It seems that about one binding site is occupied by the PEG and mPEG-anthracene on tRNA in these polymer-tRNA complexes.
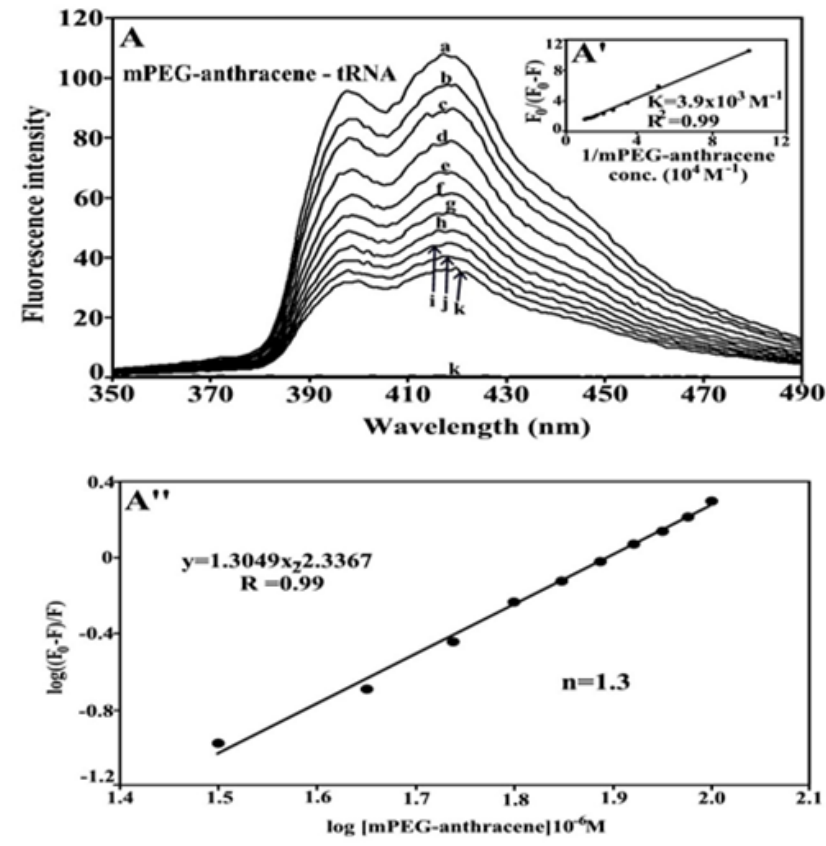

Figure 5 Fluorescence emission spectra of mPEG-anthracene-tRNA systems in $10 \mathrm{mM}$ Tris- $\mathrm{HCl}$ buffer $\mathrm{pH} 7.3$ at $25{ }^{\circ} \mathrm{C}$ for $\mathbf{A}$ ) polymer-tRNA: (a) free mPEG-anthracene $(80 \mu \mathrm{M})$, (b-k) with polymer-RNA complexes at 5 to 100 $\mu \mathrm{M}$ with (I) free tRNA I00 $\mu \mathrm{M}$. The plot of $F 0 /(F O-F)$ as a function of I/tRNA concentration. The binding constant $K$ being the ratio of the intercept and the slope for (A') mPEG-anthracene-tRNA. The plot of log (F0-F)/F as a function of log [tRNA] for calculation of number of binding sites occupied by $\mathrm{mPEG}$ anthracene molecules on tRNA $(n)$ in polymer-tRNA complexes (A").

\section{Conclusion and outlook}

In this review the bindings of several synthetic polymers with tRNA were characterized and several major points are concluded. Synthetic polymers bind tRNA through a major hydrophilic interaction and a minor hydrophobic contact. The binding is mainly through polymer polar groups $\left(\mathrm{OH}, \mathrm{NH}_{2}\right.$ and $\left.\mathrm{C}-\mathrm{O}\right)$ and tRNA bases and the backbone-phosphate group. The order of binding is PEG6000>PAMAM-G4>PEG-3350>mPEG-PAMAM-G4>mPEGPAMAM-G3>mPEG-anthracene. Synthetic polymer complexation induces major tRNA aggregation and particle formation, while tRNA remains in A-conformation. More investigations of similar nature should be done, using AFM, TEM and SEM microscopic images in order to determine the ultrastructure and morphology of synthetic polymer-RNA complexes.

\section{Acknowledgements}

This work is supported by grants from Natural Sciences and Engineering Research Council of Canada (NSERC).

\section{Conflicts of interest}

None.

\section{References}

1. Kwon YJ Before and after endosomal escape: Roles of stimuliconverting siRNA/polymer interactions in determining gene silencing efficiency. Acc Chem Res. 2012;45(7):1077-1088.

2. Knop K, Hoogenboom R D, Fischer D, Schubert US Poly(ethylene glycol) in Drug Delivery: Pros and Cons as Well as Potential Alternatives. Angew Chem Int Ed. 2010;49(36):6288-6308.

3. Zhang $\mathrm{Y}$, Chen $\mathrm{j}$, Xiao $\mathrm{C}$ et al. Cationic dendron-bearing lipids: Investigating structure-activity relationships for small Interfering RNA delivery. Biomacromolecules. 2013;14(12):4289-4300.

4. Cao Z, Jiang S Super-hydrophilic zwitterionic poly(carboxybetaine) and amphihilicnon-ionic poly(ethylene glycol) for stealth nanoparticles. Nano Today. 2012;7(5):404-413.

5. Li A, Lehmann HP, Sun G et al. Synthesis and in vivo pharmacokinetic evaluation of degradable shell cross- linked polymer nanoparticles with poly(carboxybetaine) versus poly-(ethylene glycol) surface-grafted coatings.ACS Nano. 2012;6(10):8970-8982.

6. Stiriba SE, Frey H, Haag R Dendritic polymers in biomedical applications: From potential to clinical use in diagnostics and therapy. Angew Chem Intl Ed. 2002;41(8):1329-1334.

7. Kavitha K, BhalaMurugan GL A review on PEG-ylation in anti-cancer drug delivery systems.Intl J Res Pharm Biomed Sci. 2013;4(1):296-304.

8. Di Gioia S, Conese M Polyethylenimine-mediated gene delivery to the lung and therapeutic applications. Drug Design Devel Ther. 2009;2:163-188.

9. Alexander TF Dendrimers: a versatile targeting platform. Adv Drug Deliv Rev. 2005;57(15):2104-2105.

10. Wolinsky JB, Grinstaff MW Therapeutic and diagnostic applications of dendrimers for cancer treatment. Adv Drug Deliv Rev. 2008;60(9):1037-1055.

11. Yuan Q, Yeudall WA, Yang H PEGylated polyamidoamine dendrimers with bis-aryl hydrazone linkages for enhanced gene delivery. Biomacromolecules. 2010;11(8):1940-1947.

12. Kojima C, Kono K, Maruyama K et al. Synthesis of Polyamidoamine Dendriemrs having poly(ethylene glycol) grafts and their Ability to encapsulate anticancer drugs. Bioconjugate Chem. 2000;11(6):910-917.

13. Fant K, Esbjorner EK, Jenkins A et al. Effects of PEGylation and acetylation of PAMAM dendrimers on DNA binding, cytotoxicity and in vitro transfection efficiency. Mol Pharm. 2010;7(5):1743-1746.

14. Bello-Roufai M, Lambert O, Pitard B Relationships between the physicochemical properties of an amphiphilic triblock copolymers/DNA complexes and their intramuscular transfection efficiency. Nucl Acids Res. 2007;35(3):728-739. 
15. Wang R, Zhou L, ZhouY, et al. Synthesis and gene delivery of poly(amido amines) with different branched architecture. Biomacromolecules. 2010;11(2):489-495.

16. Casettari L, Vllasaliu D, Mantovani G et al. Effect of PEGylation on the toxicity and permeability enhancement of chitosan. Biomacromolecules. 2010;11(11):2854-2865.

17. Convertine AJ, Diab C, Prieve $\mathrm{M}$ et al. $\mathrm{pH}$-responsive polymeric micelle carriers for siRNA drugs. Biomacromolecules. 2010;11(11):2904-2911.

18. Kow SC, McCarroll J, Valade D et al. Dicer-labile PEG conjugates for siRNA delivery. Biomacromolecules. 2011;12(12):4301-4310.

19. Kim W, Yamasaki Y, Jang WD et al. Thermodynamics of DNA condensation induced by poly(ethylene glycol)-block-polylysine through polyion complex micelle formation. Biomacromolecules. 2010;11(5):1180-1186.

20. Wu J, Zhou J, Qu F et al. Polycationic dendrimers interact with RNA molecules: polyamine dendrimers inhibit the catalytic activity of Candida ribozymes. Chem Commun. 2005;21(3):313-315.

21. Jensen LB, Mortensen K, Pavan GM et al. Molecular characterization of the interaction between siRNA and PAMAM G7 dendrimers by SAXS, ITC, and molecular dynamics simulations. Biomacromolecules. 2010;11(12):3571-3577.

22. De Paula D, Vitoria M, Betley LB et al. Hydrophobization and bioconjugation for enhanced siRNA delivery and targeting. RNA. 2007;13(4):431-456.

23. Bolcato-Bellemin AL, Bonnet ME, Creusat G et al. Sticky overhangs enhance siRNA-mediated gene silencing. Bolcato-Bellemin. Procced Natl Acad Sci USA. 2007;104(41):16050-16055.

24. Froehlich E, Mandeville JS, Arnold D et al. Effect of PEG and mPEG-anthracene on tRNA aggregation and particle formation. Biomacromolecules. 2012;13(1):282-287.

25. Froehlich E, Mandeville JS, Weinert CM et al. Aggregation and particle formation of tRNA by dendrimers. Biomacromolecules. 2011;12(7):2780-2787.

26. Froehlich E, Mandeville JS, Weinert CM et al. Bundling and aggregation of DNA by cationic dendrimers. Biomacromolecules. 2011;12(2):511-517.

27. Froehlich E, Mandeville JS, Arnold D et al. PEG and MPEG-anthracene Induce DNA condensation and particle formation. J Phys Chem B. 2011;115(32):9873-9879.

28. Alex S, Dupuis P FTIR and Raman investigation of cadmium binding by DNA. Inorg Chim Acta. 1989;157(2):271-281.

29. Andrushchenko VV, Leonenko Z, van de Sande H et al. Vibrational CD (VCD) and atomic force microscopy (AMF) study of DNA interactions with Cr3+: VCD and AFM evidence of DNA condensation. Biopolymers. 2002;61:243-260

30. Dovbeshko GI, Chegel VI, Gridina NY et al. Surface enhanced IR absorption of nucleic acids from tumor cells: FTIR reflectance study. Biopolymer. 2002;67(6):470-486.

31. Ahmed Ouameur A, Tajmir-Riahi HA Probing tRNA interaction with biogenic polyamines. RNA. 2010;16(10):1968-1979.
32. Marty R, N' soukpoe-Kossi CN, Charbonneau D et al. Structural characterization of cationic lipid- tRNA complexes. Nucl Acids Res. 2009;37(15):5197-5207.

33. Ahmed Ouameur A, Tajmir-Riahi H A Structural analysis of DNA interactions with biogenic polyamines and cobalt (III) hexamine studied by Fourier transform infrared and capillary electrophoresis. $J$ Biol Chem. 2004;279(40):42041-42054.

34. Froehlich E, Jennings CJ, Sedaghat-Herati MR et al. Dendrimers bind human serum albumin. $J$ Phys Chem B. 2009;113(19):6986-6993.

35. Kypr J, Vorlickova M Circular dichroism spectroscopy reveal invariant conformation of guanine runs in DNA. Biopolymers. 2202;67(4-5):275-277.

36. Vorlickova M Conformational transitions of alternating purinepyrimidine DNAs in the perchlorate ethanol solutions. Biophys $J$. 1995;69(5):2033-2043.

37. Connors K Binding constants: The measurement of molecular complex Stability. John Wiley \& Sons, New York. Pp. 432. 1987

38. Maiti PK, Caing T, Wang G et al. Structure of PAMAM dendrimers: Generations 1 through 11. Macromolecules. 2004;37(16):6236-6254.

39. Patri AK, Majoros IJ, Baker Jr JR Dendritic polymer macromolecular carriers for drug delivery. Curr Opinion Chem Biol. 2002;6(4):466-471.

40. Del Valle J, Turek AM, Tarakalano D, Saltiel J Distortion fluorescence spectrum of anthracene with increasing laser puls excitation energy. $J$ Phys Chem A. 2002;106(20):5101-5104.

41. Zheng Y, Micic M, Mello SV et al. PEG-based hydrogel synthesis via the photodimerization of anthracene groups. Macromolecules. 2202;35(13):5228-5234.

42. Lakowicz JR Principles of Fluorescence Spectroscopy (3nd edn), Springer, New York. 2006

43. Dubeau S, Bourassa P, Thomas TJ et al. A Biogenic and synthetic polyamines bind bovine serum albumin. Biomacromolecules. 2010;11(6):1507-1515.

44. Mandeville JS, N'soukpoé-Kossi CN, Neault JF et al. Structural analysis of DNA interaction with retinol and retinoic acid. Biochem Cell Biol. 2010;88(3):469-477.

45. Charbonneau DM, Tajmir-Riahi HA Study on the interaction of cationic lipids with bovine serum albumin. $J$ Phys Chem B. 2010;114(2):1148-1155.

46. Mandeville JS, Tajmir- Riahi HA Complexes of dendrimers with bovine serum albumin. Biomacromolecules. 2010;11(2):465-472.

47. N'soukpoé-Kossi CN, Bourassa P, Mandeville JS et al. Modelling of vitamin A binding to tRNA. J Pharm Biomed Anal. 2014;99:28-34.

48. Agudelo D, Bourassa P, Beauregard M, Berube' G, Tajmir-Riahi HA tRNA binding to antitumor drug doxorubicin and its analogue. PLoS ONE. 2013;8(7):e69248.

49. Bourassa P, Thomas TJ, Bariyanga $\mathrm{J}$ et al. Breast anticancer drug tamoxifen and its metabolites bind tRNA at multiple sites. Int $J$ Biol Macromol. 2015;72:692-698. 